\title{
Significados docentes sobre violencias en las relaciones laborales en la escuela ${ }^{1}$
}

\author{
Mg. Pamela Barría Herrera² \\ Dr. Christian Miranda Jaña ${ }^{3}$ \\ Dra. María Isabel Toledo Jofré 4 \\ Mg. Gabriel Guajardo Soto ${ }^{5}$
}

\begin{abstract}
RESUMEN
El estudio busca comprender los significados de profesores sobre violencias en las relaciones laborales en la escuela. A partir de un enfoque socio-histórico y metodología sustentada en la etnográfica interpretativa, se realizan observaciones y entrevistas en tres escuelas de la Región Metropolitana; éstas son analizadas mediante el procedimiento de descripción densa. Los resultados por dimensión destacan que: i) en los "contratos individuales" se presenta mayor trabajo real que el prescrito; ii) en las "relaciones sociales" son constantes los episodios de violencia verbal; iii) en las "relaciones colectivas" se observa limitada participación de docentes en la toma de decisiones, y iv) en lo referente a "normativas", en la escuela de dependencia municipal hay mayor impacto de las políticas educativas, mientras las normas internas afectan en mayor medida en la escuela privada. Se concluye que los factores observados en las dimensiones del concepto relaciones laborales, favorecen la intensificación laboral y malestar docente.
\end{abstract}

Palabras clave: Violencia en la escuela, significados docentes, relaciones laborales, profesores, etnografía.

\section{ABSTRACT}

This study tries to understand the meanings of teachers about violence in school labor relationships. From a socio-historical approach and using methodology based on ethnographic interpretation, observations and interviews are applied in three schools located in the Metropolitan Region; these are analyzed through the dense description procedure. Results by dimension emphasize that: i) "individual contracts" represent more real work than the prescribed one; ii) in "social relationships", episodes of verbal violence are frequently experienced; iii) in "collective relationships", teachers participation in decision-making processes is limited, and; iv) concerning to the school "norms", educational policy has greater impact in public schools, while internal rules have greater impact in private schools. It is

I Estudio realizado en el marco de los Proyectos Fondecyt $\mathrm{N}^{\circ}$ II $40135 \mathrm{y} \mathrm{N}^{\circ}$ II 70328 financiados por CONICYT-Chile.

2 Magíster en Psicología Educacional. Profesora de la Universidad Metropolitana de Ciencias de la Educación. E-mail: Pamela. barria@ug. uchile.cl

3 Doctor en Ciencias de la Educación. Profesor Asociado del Departamento de Educación, Universidad de Chile. E-mail: Christian. miranda@u. uchile.cl

4 Doctora en Educación. Profesora Titular de la Escuela de Psicología, Universidad Diego Portales. Chilena.maria.toledo@udp.cl

5 Magíster en Psicoanálisis. Investigador Asociado de la Facultad Latinoamericana de Ciencias Sociales, FLACSO-Chile. E-mail: gabriel. guajardo@flacsochile. org 
concluded that the factors observed in the dimensions of the labor relationships concept, promote labor intensification and teacher uneasiness.

Keywords: Violence in School - meanings of teachers - labor relationships - Teachersethnography.

Cuando una conducta es señalada como violenta o potencialmente violenta, es inmediatamente entendida como una conducta peligrosa, pues compromete a la sobrevivencia y la defensa de la sociedad contra todas las amenazas (Dubet, 2003). En concordancia a ello, las violencias en la escuela son una temática actual y preocupante en los sistemas educativos contemporáneos, pues se han visto sus efectos nocivos (Berger y Lisboa, 2012).

Si bien los marcos reguladores que rigen el trabajo del docente, como el Estatuto Docente y la Ley de Calidad y Equidad de la Educación, abordan el derecho de los profesionales de la educación a trabajar en un ambiente de tolerancia, de respeto mutuo a su integridad física, psicológica y moral por parte de los integrantes de la comunidad educativa (Ley 19.070, 1997; Ley 20.50I, 201 I), poco se ha reportado en la literatura sobre la violencia que viven los docentes, y cuando se hace, solo se describen interacciones violentas entre directivos y docentes (Blasé \& Blasé, 2002) y entre colegas (OrtizMolina, 20II).

Entendiendo la labor del docente como un trabajo, un conjunto de tareas y relaciones humanas estructuradas de determinadas maneras, ubicadas en la escuela como espacio físico (Hargreaves, 2003), es que resulta pertinente abordar las violencias en el trabajo docente desde el concepto de relaciones laborales. Éstas "comprenden las instituciones, reglas y normas que regulan la vida social en la empresa y economía general, la interacción entre actores individuales y colectivos" (Kohler y Artiles, 20I0, p. 56). Engloban relaciones entre distintos actores - personas, grupos u organizaciones- que generan variadas formas de regulación-normas, instituciones, reglas informales-. Las relaciones laborales se articulan en cuatro dimensiones interdependientes: contrato individual, relaciones sociales en el trabajo, relaciones laborales colectivas y normativa laboral (Kohler y Artiles, 2010). 
Se identifica una brecha de conocimiento respecto a lo que sucede en el trabajo del docente en la escuela frente al fenómeno de las violencias, por lo que este estudio pretende contribuir con conocimiento social a partir de la comprensión de las violencias en las relaciones laborales en establecimientos educativos de la Región Metropolitana de Chile. A partir de ello, se define como objetivo del estudio comprender las violencias en las relaciones laborales en la escuela, a partir de los significados de profesores y profesoras.

Las violencias son un factor que afecta la calidad de vida laboral y el desarrollo de la enseñanza (Hargreaves, 2003); cobra relevancia tratar este fenómeno como un aporte al trabajo docente y convivencia escolar, asumiendo que la construcción de un buen trato en la comunidad escolar involucra a los profesionales de la educación, quienes tienen por función favorecer la construcción de un ambiente propicio para el aprendizaje.

A partir de lo anterior, este trabajo se estructura en torno a tres ejes de desarrollo. El primero, aborda las premisas conceptuales y metodológicas que guían la comprensión del fenómeno de la violencia en las relaciones laborales. El segundo, donde se presenta de modo sintético los principales hallazgos del estudio, y el tercero, donde se concluye respecto del trabajo, focalizando en las implicancias educativas del estudio.

\section{Antecedentes}

Definir violencias resulta una tarea compleja, pues existe diversidad de paradigmas y perspectivas para abordarla (García y Madriaza, 2005; Berger y Lisboa, 2012). Así, existen múltiples tipos de violencias, que varían en intensidad de sus prácticas, finalidades y naturaleza; además es relativa, según épocas, medios sociales y culturales, siendo entonces, necesario hablar de "las violencias" y no de "la violencia" (Crettiez, 2009).

Se comprenderá el fenómeno, desde la expresión "Violencia en la Escuela”. Fernández, Pencacho y Candelas (201 I), a partir de los planteamientos de Dubet (2005), la distinguen como aquellas violencias de los actores, como estudiantes, profesores, apoderados, 
entre otros. Así, tiene la ventaja de ampliar el fenómeno entre distintos miembros de la comunidad educativa (Zerón, 2006). En armonía con lo anterior se comprende la violencia en la escuela como:

La desorganización brutal o continua de un sistema personal, colectivo o social traduciéndose por una pérdida de integridad que puede ser física, psíquica o material. Esta desorganización puede operarse por agresión, uso de la fuerza, conscientemente o inconscientemente, pero puede haber violencia, del punto de vista de la víctima, sin que haya necesariamente agresor ni intención de dañar. La violencia es dependiente de los valores, códigos sociales y fragilidades personales de las víctimas. (...) estudiar la violencia en el medio escolar es encaminarse hacia una reflexión sobre el sentido mismo de las prácticas educativas (Debarbieux, 1996, pp. 45-46).

Las violencias son una forma de relación entre las personas que es percibida y atribuida por quienes la viven (Valdivieso, 2009); es un conjunto de conductas, un síntoma y un objeto político sobre el cual pueden construirse amplias unanimidades ideológicas, sindicales, profesionales y políticas (Dubet, 2003).

Se observan redes causales asociadas a factores internos y externos de la escuela. Los primeros, abordados en este trabajo, corresponden a factores estructurales como por ejemplo el número de estudiantes por aula, o pedagógicos, como la estabilidad de los equipos docentes (Debarbieux \& Blaya, 2009). En la misma línea, la instalación del modelo neoliberal en el sistema escolar ha transformado las condiciones del trabajo docente, produciendo intensificación del trabajo, estandarización, cuestionamiento de la identidad profesional y deterioro de los ambientes laborales (Cornejo, 2007), lo que va aparejado a la descentralización del sistema escolar y su privatización (Assael, Cornejo, González, Redondo, Sánchez \& Sobarzo, 20I I). A partir de ello, los establecimientos escolares chilenos operan bajo tres tipos de administración: escuelas estatales, gestionadas por el municipio; particulares subvencionadas, gestionadas por sostenedor privado con subsidios estatales; particulares privadas, gestionadas por un sostenedor privado sin subsidio estatal (Cornejo, Albornoz, 
Castañeda, Palacios, Etcheberrigaray, Fernández, Gómez, Hidalgo \& Lagos, 2005).

Desde la literatura, hay estudios que abordan las violencias en la escuela en las relaciones laborales. En Estados Unidos, a partir de una encuesta nacional de victimización docente desarrollada por APA Classroom Violence Directed Against Teachers TaskForce, se constata un conjunto de agresiones realizadas de parte de colegas a docentes. En efecto, 24\% sufrió intimidación, I6\% fue víctima de internet y I I \% vivió ataque físico con visita al médico (McMahon et al., 20I4). En el ámbito de violencia desde directivos a profesores, los resultados de encuestas realizadas a docentes de escuelas primarias, intermedias y secundarias, señalan cinco comportamientos de maltrato frecuentes: no reconocer o alabar logros relacionados con el trabajo (70\%), «otros» maestros favorecidos (63\%), intento de intimidación (59\%), no apoya en interacciones difíciles con estudiantes y/o padres (57\%), e ignorar o hacer desaires (55\%) (Blase \& Blase, 2007).

Ortiz-Molina (20II) estudia las relaciones de violencia entre profesores y desde directivos hacia docentes en Bogotá-Colombia. En sus resultados, señala que la violencia psicológica no es percibida como grave, pero afectan en la salud mental con el desarrollo de enfermedades en este ámbito. Otro hallazgo es que los conflictos con directivos son percibidos como interacciones negativas cuando la actitud es de desconocimiento e irrespeto al maestro, por ejemplo cuando se ignora la palabra del otro.

En Chile, el Ministerio de Educación el año 2005 aplica una encuesta sobre la violencia escolar a estudiantes y docentes. Entre sus resultados, evidencian que un $32 \%$ declaran haber sido agredidos en la escuela, de los cuales un $27 \%$ corresponde a hombres y $34 \%$ a mujeres. Ahora bien, $8 \%$ señala haber sido agredido por otro profesor, mientras que existe prevalencia de las agresiones psicológicas (47\%).

Mientras, en Valparaíso se desarrolló un estudio sobre victimización de profesores. Entre sus resultados, un I7\% de docentes indicó haber sido ridiculizado o humillado por un par en el último mes y $35 \%$ señaló haberlo observado en otro docente. II\% vio a un docente amenazando a otro con herirlo. Al indagar sobre las violencias 
desde directivos a docentes, 17\% de informantes reportan haber sido ridiculizado o humillado por un directivo de su escuela al menos una vez durante el último mes y $9 \%$ fue amenazado. $27 \%$ de los docentes indicó haber visto a otro profesor ser ridiculizado o humillado por un integrante del equipo directivo (Morales, Álvarez, Ayala, Ascorra, Bilbao, Carrasco, López, Olavarría, Ortiz, Urbina, y Villalobos, 2014).

Por otro lado, cuando se identifican las condiciones contractuales de profesores en Chile, éstos declaran que el 68,5\% tiene jornada completa y el 62,9\% tiene contrato indefinido (Mineduc, 2013). En la misma línea se sitúa un estudio de Unesco (2005), el cual plantea que un $20 \%$ de profesores encuestados en Chile tienen un trabajo adicional al de docente. En una escala de medición de la carga de trabajo que se realiza fuera del horario laboral, sobre la base de diez tareas (preparar clases, aplicar evaluaciones, atención de padres y alumnos, planificación, entre otras), prácticamente el $50 \%$ de docentes encuestados se encuentra sobre los seis puntos, es decir, realizan seis o más tareas relacionadas con su trabajo fuera del horario asignado. Las principales tareas que se realizan fuera del horario de trabajo son: preparación de material didáctico (87\%), cursos de perfeccionamiento (86\%) y preparación de clases (83\%).

En suma, la evidencia da cuenta de la existencia del fenómeno de violencia hacia los docentes en su contexto laboral. En ella, se observan diferentes formas que asume y actores educativos que participan. En el caso de Chile, hay predominancia en declarar violencia de carácter psicológico, sin diferencias significativas según género. Sin embargo es necesario recalcar las condiciones laborales que se presentan en el trabajo docente, como una manifestación de violencia.

Por último, el estudio se basa en el concepto de significados desde Brunner (1990), autor que lo aborda desde un enfoque sociohistórico. El concepto se desarrolla asumiendo un origen biológico y cultural que se entrecruzan por medio de la negociación. En lo cultural, se encuentran inmersos los sistemas simbólicos en los que se construye el significado de las cosas. Así, para la comprensión de las personas, es necesario comprender sus experiencias y actos moldeados 
por sus estados intencionales. La forma de esos estados sólo puede plasmarse mediante la participación en los sistemas simbólicos de la cultura. Así, la forma de vida es comprensible en virtud de los sistemas culturales de interpretación, adquiriendo el significado una forma pública y comunitaria, arraigado al lenguaje y la cultura.

\section{Metodología}

La investigación se sitúa desde un método cualitativo con uso del enfoque interpretativo-comprensivo, ya que busca comprender el fenómeno de las violencias en las relaciones laborales en la escuela desde los propios actores (Mucchelli, 1996). A partir de la etnografia, se aborda la unidad social de la escuela, realizando una descripción e interpretación de grupos sociales, en este caso profesores, a través de los patrones observables y aprendidos del comportamiento de tales grupos (Govea, Vera y Vargas, 20II). Específicamente se procede a utilizar los planteamientos de la etnografía interpretativa, entre cuyos rasgos característicos está la interpretación de discursos sociales intentando "rescatar lo dicho en ese discurso de sus ocasiones perecederas y fijarlo en términos de consulta” (Geertz, 2005:32).

Las instituciones educativas participantes en el proceso investigativo son representantes de los distintos tipos de dependencias educativas chilenas: municipales, subvencionadas y privadas. Estas se ubican en 3 comunas de la zona urbana de la Región Metropolitana, cuya selección considera distintas realidades sociales, según el Índice de Desarrollo Humano [IDH]. Todas las escuelas coinciden en ser mixtas y brindar educación básica. Además, el estudio considera la participación de profesores de educación básica que realizan sus labores profesionales en el establecimiento por un periodo mínimo de I año. Se incluyen a encargados de Convivencia y Directivos docentes dada su relevancia y participación en el tema.

En este caso, el problema de investigación se centró en la mirada de los profesores y profesoras sobre la violencia en las relaciones laborales, esto a través de las significaciones de los actores frente al fenómeno. El proceso etnográfico se llevó a cabo mediante observaciones y entrevistas. Se obtiene un total de 5 observaciones en consejo de 
profesores o instancia similar, 4 en el establecimiento municipal y I en el de administración privada, cuyo registro se realiza por medio de notas de campo y audio. En el caso de las entrevistas, se efectúan un total de 25 entrevistas, de las cuales II corresponden al colegio municipal, 5 en establecimiento subvencionado y 9 en privado; todas ellas registradas por medio de grabadoras de audio y luego transcritas a texto.

Los datos obtenidos fueron sometidos a análisis de acuerdo a lo planteado por el enfoque, es decir bajo descripciones densas, proceso que corresponde a cierto tipo de esfuerzo intelectual, una especulación elaborada (Govea, Vera y Vargas, 201 I). De esta forma, el análisis etnográfico consiste en desentrañar las estructuras de significación y en determinar su campo social y su alcance (Geertz, I992), metas definidas en los objetivos de esta investigación.

\section{Resultados}

A continuación se presentan los resultados alcanzados en el proceso etnográfico, los cuales son abordados a partir de las dimensiones que componen el concepto de relaciones laborales: contrato individual, relaciones sociales en el trabajo, relaciones laborales colectivas y normativa laboral (Kohler y Artiles, 2010).

\section{Contrato Individual}

Los contratos individuales se establecen entre dos partes: profesores y la figura del sostenedor. Este último, de acuerdo al tipo de dependencia, puede ser municipio o sostenedor particular; en el caso del colegio privado, a partir de la figura de gerencia.

Es coincidente entre los establecimiento educacionales, la limitada distribución de horas no lectivas frente a las lectivas. Ejemplo de ello, en el colegio privado "profesores de educación media, como en inglés, tienen 42 boras... son 42 boras frente al curso" (PCC). En ese sentido, las horas destinadas para trabajo no lectivo en el establecimiento son ausentes o limitadas respecto al trabajo requerido: "Lamentablemente todas las horas que bace en el colegio para planificar no son suficientes y atender apoderados, casos 
especiales, corregir. Todo eso no alcanza en el colegio. Esos 15 minutos que tenemos por cada bora. Todo ese trabajo que no alcanza, lo tiene que llevar para la casa" (PSI). El establecimiento educacional privado cuenta con un contrato de servicios, el cual tiene como objetivo velar por los servicios prestados, brindando protección y posición de cliente-contratante al apoderado, pero vulnera el rol docente en la práctica cotidiana, ya que se pueden enfrentar a acciones judiciales ante la posible falta, es decir, incumplimiento de contrato. De esta manera, este tipo de contrato no actúa como un aspecto protector de su labor, sino más bien, perciben vulnerabilidad. Situación similar ocurre en el colegio subvencionado, pues los apoderados se posicionan con rol del clientecontratante. Al pagar por un servicio, se exige a quienes trabajan en el espacio educativo, principalmente a profesores e inspectores, con solicitudes que en ocasiones van más allá de los límites educativos. Esta situación es imitada por estudiantes, quienes reiteran el discurso de exigencia en virtud de ser quienes pagan: "acá el apoderado es muy violento en eso. Te cobran. Estoy pagando, ustedes me tienen que criar al bijo" (SP2).

En el caso del colegio municipal, se identifica que, para el desarrollo de un mismo trabajo, existen diferentes tipos de contratos: titularidad, contrata y por ley SEP. La titularidad es un tipo de contrato que presenta mayores garantías, ya que se basa en el estatuto docente; presenta mayor seguridad en la continuidad laboral y permite participar en acciones del gremio. Sin embargo, no hay certeza de que se mantengan las horas asignadas por contrato de parte del sostenedor, lo que genera inseguridad laboral y desconfianza. En segundo lugar, se encuentran los docentes "a contrata", cuyo contrato es definido en el tiempo con posibilidades de renovación; este tipo de contrato no cuenta con certeza de su continuidad laboral. El tercer tipo de contrato es aquel cuyas labores se enmarcan bajo la ley SEP; es un tipo de contrato en el cual no hay certeza en la continuidad laboral y se encuentra bajo los lineamientos del código del trabajo. Un porcentaje mayor de profesores del establecimiento educacional se encuentran en esta modalidad.

La relación entre matrícula y puesto de trabajo es una relación de dependencia dramática dentro de la rutina escolar, pues se percibe como amenaza constante. La comunidad genera acciones para la 
retención y captación de matrículas, lo que afecta en los tiempos destinados a labores pedagógicas. "La matrícula en otros (tipos de dependencia) no es una preocupación para el profesor y acá sî" (MP3).

\section{Relaciones sociales en el trabajo}

Es coincidente entre los tres colegios estudiados, que sus profesores se congregan en grupos: en el caso del colegio particular, por ciclo escolar; en el colegio subvencionado, por disciplina, y en el colegio municipal, debido a antigüedad, realizando distinción entre los que ingresaron en este último periodo y los que llevan más años en ejercicio en el colegio. Esta forma de agrupación se encuentra normalizada por sus profesores. Se presenta limitada interacción inter-grupo, pero una buena o adecuada relación intra-grupo en los tres escenarios.

Dentro de las interacciones, en el colegio privado son violentas aquellas acciones relacionadas con el menoscabo, realizado de manera verbal, a partir de actos denominados cahuines o pelambres - hablar mal del otro-. Otra manifestación es la exclusión de colegas en las relaciones sociales cotidianas, al apartar de la interacción en los espacios comunes. Lo anterior se refleja en la siguiente cita: "una profesora nueva que llegó este año, al principio le bicieron vacío, no la invitaban a los almuerzos"(PI), pues "la encontraban rasca... que era cbula" (PPS). El concepto de exclusión es un fenómeno normalizado, en el cual la afinidad es el factor que sustenta la vinculación entre profesores.

Como causas de las violencias entre docentes se basan, en primer lugar, en celos profesionales debido a las diferencias percibidas entre los grupos constituidos por nivel escolar y sus diferentes estilos de trabajo. En segundo lugar, se observa la categorización de docentes por comportamientos asociados a clases sociales consideradas diferentes. En tercer lugar, se plantea la presencia de envidia hacia aquel que se destaca; las diferencias son devaluadas y responsables de las violencias en las relaciones. Se atribuye como raíz de este fenómeno, el impacto de la dictadura militar en la historia nacional y el aislamiento territorial como país, que afectó en el acceso para conocer distintas realidades. 
En las relaciones entre directivo y profesores, estos últimos identifican verticalidad y menor red de contención en el trabajo, pues desde los docentes, plantean que quien se desempeña como directivo no visualiza las necesidades, complejidades y rutinas de trabajo de sus profesores. Se define a la dirección como figuras sancionadoras, duras, distantes, ausentes y autoritarias. En la comunicación entre las partes, directivos y docentes, se observan estrategias de evitación y disgregación para abordar temáticas atingentes a la vida cotidiana en el establecimiento educacional. Ejemplo de ello se refleja en la siguiente cita: "Teníamos muchísimos problemas, entonces decían: no, boy bay trabajo administrativo; cuando babía una norma dura, decían: no, boy tienen una capacitación"(PP).

En el colegio subvencionado, las relaciones intra grupo se consideran como adecuadas, sin embargo intergrupo se manifiestan como rudas, distantes y tensas. Las formas de la violencia entre docentes, ocurren de manera verbal, a través de insultos. Los motivos se deben a diferencias en el ámbito de trabajo o por problemas de comunicación; además aluden a la idiosincrasia chilena y el impacto de la dictadura militar, la cual, como un episodio histórico, presenta complejas influencias en las relaciones sociales de los actores en el colegio. Entre sus manifestaciones se identifica como herencia la intolerancia a la opinión que difiere del consenso, la desconfianza en quien lo rodea y el desarrollo de la competitividad, pues "Ese es el país que tenemos, todo tiene que ser consenso, tú no puedes discrepar del otro. Si discrepas estás mal... Abí nuevamente podemos ir al tema de la dictadura... Los que crecimos con dictadura, desconfiados y competitivos" (SP2).

En el establecimiento educacional municipal se identifican las bromas como un elemento presente y valorado en la interacción de los docentes de este establecimiento, pues daría cuenta de un ambiente distendido entre los miembros. La confianza y el criterio son requisitos para hacer una broma, sin embargo, no consideran la recepción del otro, observando malestar, enojo y queja en su persona. Aquello que se menciona como broma, tiende a abordar temáticas como hechos ocurridos en las prácticas cotidianas, imagen corporal y género de manera denotativa hacia miembros de la comunidad. Por ello se configuran como un recurso en el que se abordan temas que no se dicen directamente. 
La relación entre directivos y docentes es vertical: el equipo directivo castiga el incumplimiento de tareas, llama la atención y genera orden ante los comportamientos no esperados dentro de las dinámicas instituidas. De parte de la dirección, hay un discurso de escuchar el parecer de los docentes, sin embargo, se desconocen los aportes del profesor bajo el argumento de diferenciar la experiencia. En cuanto a las relaciones entre profesores y equipo directivo, se manifiesta como causa de conflicto no seguir lo establecido de parte del equipo directivo, como seguir horarios.

\section{Relaciones colectivas}

En el establecimiento educacional privado, hay convenios colectivos y un sindicato de docentes. Esta agrupación en conformación no agrupa a la totalidad de profesores del establecimiento educacional. Es una organización que no tiene incidencia suficiente en las relaciones laborales de sus trabajadores ni en las necesidades identificadas por los mismos docentes. De este modo, el sindicato como organización aún no cumple sus objetivos de resguardar las condiciones laborales.

En el caso del establecimiento subvencionado, hay prohibición de acciones colectivas como el desarrollo de sindicato; sin embargo, hay negociaciones colectivas las cuales son limitadas en el tiempo y a beneficios acotados a aspectos monetarios por sobre condiciones laborales para la comunidad educativa.

En el colegio municipal hay una agrupación gremial de docentes en directa relación con el colegio de profesores. Se encuentra legitimada en la escuela al contar con espacios de participación; sus objetivos son conocidos por los docentes, pues recurren a ellos como un espacio de organización colectiva que brinda amparo ante las necesidades que ocurren en el trabajo docente. Hay participación parcial, la que ha disminuido en el tiempo. Los motivos por los cuales se presenta una baja participación en espacios colectivos se centran en las dificultades para reunirse por el tiempo y a la inseguridad de continuidad laboral condicionada por los tipos de contratos.

Participar de acciones en el marco de las relaciones colectivas ha conllevado la amenaza de castigos en el establecimiento municipal. 
Una docente señala: "me mandaron sola a otra escuela donde no me querían recibir porque decían que yo estaba en la lista negra" (MP2). De este modo, se da cuenta que entre las amenazas se encuentran cambios de escuela en la misma comuna, sumarios por abandono de trabajo, pérdida del empleo, temor y evaluación de descuentos en remuneraciones; no todas ellas se concretizan, al llegar a negociaciones con el sostenedor. En este aspecto es donde se hacen más patentes las diferencias de contrato, las cuales limitan la participación al no contar con certezas laborales.

Según lo observado, la participación de profesores en espacios de trabajo como consejos de profesores, es pasiva; se limitan a la recepción de información y decisiones tomadas por otros agentes de la escuela. Ejemplo de ello se representa en la siguiente cita: " $L a$ mayoría este año ba sido informativa, más que nada. Resolutivo no bemos tenido ninguno basta el momento" (SPI). En el caso del colegio privado, se observa que la Dirección tiene una posición de acatamiento hacia las decisiones tomadas por la gerencia, lo que grafica la verticalidad de relaciones entre los distintos estamentos que conforman esta sociedad educacional.

\section{Normativas}

En lo referente a normativas laborales, en los establecimientos privados y particulares subvencionados relevan aquellas normas de carácter interno. Ejemplo de ello es el uso del uniforme institucional como mecanismo de disciplinamiento. En el primer tipo de establecimiento, se marca la diferencia entre roles al flexibilizar su uso en aquellos que presentan mayor jerarquía; a su vez ocurre resistencia por docentes jóvenes.

En el establecimiento privado, hay normativas internas que afectan en el control del tiempo de sus profesores, como el almuerzo, o aplicando la burocracia como manifestación de desconfianza ante la participación del docente en sus tiempos de trabajo. La manifestación de resistencia hacia la norma es pasiva, al dejar de seguirlas en el transcurso el tiempo.

En el caso del colegio municipal, toman mayor lugar las normas externas, las cuales se posicionan como fuentes de tensión y 
sobrecarga laboral. Ellas se conforman a través de las Políticas Educacionales, como el proyecto de integración escolar [PIE] y la Ley de Subvención Escolar Preferencial [SEP]. Ambos, si bien son programas de apoyo, se convierten en una instancia burocrática, que exige variados medios de verificación que acotan el tiempo de trabajo en acciones de rendición de cuentas. De esta manera señalan: "Hay bartas cosas que nosotros bacíamos, pero no lo tenemos escrito, que la agencia lo solicita. Hay que bacer protocolos. Hay que hacerlos más sistemáticos, con los papeles al día, tener todo muy documentado" (MOB).

El establecimiento educacional convive con distintas evaluaciones externas. Una de ellas es la medición realizada por el SIMCE en aspectos de rendimiento académico y aspectos de convivencia escolar. Para el trabajo docente es una presión ante las categorizaciones resultantes, más aún cuando el desempeño es señalado como deficiente, ya que se transforma en una amenaza de intervención del Ministerio de Educación.

La Evaluación Docente responde a una política que para profesores les significa violencia y estrés, debido a las condiciones en las que esta se desarrolla. Se convierte en otro factor de sobrecarga laboral, ya que no se consideran los tiempos de trabajo extra que se requieren para cumplir con las exigencias planteadas, como, por ejemplo, el desarrollo del portafolio. Esta sobrecarga se traduce en la necesidad de ocupar espacios de tiempo personal y de descanso.

Se hace evidente en la escuela municipal el papel de instituciones que supervisan el trabajo en la escuela. Una de ellas es la Agencia de Calidad, cuya supervisión contempla tiempo de permanencia en la escuela para la observación de su dinámica. Este proceso finaliza con la categorización de colegios, lo que para el establecimiento educacional es visto como "no punitivo". En cambio, la visita de la Superintendencia de Educación al establecimiento es definida como punitiva al hacer uso de multas ante el error, por ello, genera tensión. Situación similar ocurre ante la visita de Subvención escolar, instancia abocada a la asistencia y pago de la misma. También es punitiva ante el error, generando altas multas en dinero. Las organizaciones que visitan la escuela, finalmente, se convierten en sistemas de control basados en la desconfianza de parte de 
las organizaciones del Ministerio de Educación frente al trabajo realizado en la escuela.

Estas supervisiones generan tensión en la comunidad educativa, pues en el cotidiano se reiteran las indicaciones a los docentes con el objetivo de evitar sanciones y se amenaza con castigos como registrar en la hoja de vida alguna posible falta o incumplimiento. Para los docentes, "la impresión que nosotros tenemos es que la gente que viene a supervisarte en ese aspecto, viene pensando que tú bas becho algo indebido, viene predispuesto, esa es la impresión que tiene uno, de pillarte en algo, entonces indudablemente eso te tensiona" (MUTP).

Por último, las diferencias percibidas entre lo que exige el Ministerio de Educación como metas y la realidad de los procesos de aprendizaje de estudiantes en una escuela vulnerable. Ello se debería al bajo conocimiento de la realidad de un establecimiento educativo categorizado como tal. Como consecuencia, esta diferencia sobrecarga al docente.

\section{Conclusiones}

Este estudio se basa en la experiencia de aproximación etnográfica vivenciada en tres establecimientos educacionales de la Región Metropolitana, cada uno de los cuales corresponde a las diversas dependencias: municipal, particular y subvencionado. En los tres establecimientos se han evidenciado violencias en las relaciones laborales de docentes, en distintas manifestaciones.

Al describir violencias en las relaciones laborales desde ámbitos normativos y contractuales, es transversal el impacto de la distribución de horas, respecto a las tareas lectivas-no lectivas; esto da cuenta de la invisibilización de la complejidad de la labor docente en la práctica cotidiana, la cual no se limita al aula.

Es coincidente entre el colegio privado y particular subvencionado, que profesores evidencien violencias en su relación con el apoderado. Estas se deben a que esta figura es posicionada como cliente-contratante. En base a ello, los apoderados hacen reclamos y demandas de necesidades en un status similar a elementos de consumo y, por ende, sus dinámicas 
adquieren similitud a otro espacio de venta. Es una problemática identificada como propia de estos tipos de dependencia.

En el caso del establecimiento municipal, sus profesionales de la educación perciben violencia en los distintos tipos de contrato que toman lugar para una misma labor, los cuales se sustentan desde distintas normativas laborales. La continuidad laboral y la gestión de horas son elementos en los que también se vivencia la violencia, ya que puestos laborales de que dependen principalmente desde la matricula alcanzada anualmente. Por ello, la retención y captación de matrículas son actividades que se incorporan en sus prácticas laborales, afectando en el desarrollo de su trabajo pedagógico.

Frente a los aspectos normativos, en los establecimientos privados y particulares subvencionados, destaca la presencia de normas internas que tienen como objetivo el disciplinamiento de los docentes. En cambio, en el establecimiento municipal, toman mayor protagonismo desde los significados de los profesores, aquellas normativas que provienen desde el exterior a la escuela, conformadas a través de la ejecución de las políticas educativas. Ejemplo de ello es la sobrecarga laboral frente a la rendición de cuentas a laque se somete la comunidad educativa, ya que la documentación es un elemento indispensable para las constantes supervisiones a las cuales son sometidos; este procedimiento es visto como desconfianza del trabajo realizado por la escuela y tiene consecuencias como categorizaciones o multas. Los profesores son conscientes de las violencias vistas bajo los procesos de evaluación, las que tienen como consecuencia sobrecarga laboral, presión y la invisibilización de las necesidades de los docentes.

Al describir las violencias en las relaciones interpersonales y colectivas, se visualiza en los tres establecimientos, la tendencia a relacionarse en grupos cuyos criterios para conformarlos apuntan a elementos formales. Sin embargo las relaciones intergrupos tienden a presentar mayores roces. En el cotidiano, la violencia interpersonal es principalmente verbal, como en el acto de hablar de otras personas en forma de menoscabo, la exclusión del grupo, insulto, bromas, descalificaciones, sobrenombres e ironías. Las razones por las cuales ocurren, se depositan en la idiosincrasia, la historia de Chile, la envidia y postura alcanzada 
ante la diferencia -como característica personal o en la forma en que realizan su trabajo-. Entre directivos y docentes, es coincidente que entre ambos actores identifican la verticalidad como parte de sus relaciones.

Las relaciones laborales colectivas difieren entre los establecimientos, ya sea por la prohibición para el desarrollo de sindicatos; al encontrarse en formación;o bien, al identificarse como una instancia de apoyo a quienes son colegiados en el caso de la escuela municipal; a pesar de ello, en el mismo establecimiento se evidencian sanciones de parte del sostenedor a la participación en movimientos colectivos. Es coincidente en los establecimientos una participación pasiva de profesores en espacios de consejo o similares, limitándose a escuchar las decisiones tomadas por sostenedores.

A través de los resultados alcanzados, se identifican distintas manifestaciones de violencias en las relaciones laborales: una de ellas es la violencia simbólica, la cual se caracteriza por ser ausente de dolor visible pero culturalmente legitimado, la cual opera bajo un mecanismo de "servidumbre voluntaria" (Bourdieu, y Wacouant, 1995; Crettiez, 2009). Ello toma lugar en los distintos tipos de contrato para una misma tarea y las diferencias del trabajo real y prescrito que limita el trabajo solo al aula, invisibilizando lo no lectivo. También sucede ante la prohibición y castigo de la actividad sindical, el disciplinamiento del docente, las supervisiones y evaluaciones a la comunidad educativa.

Se desarrollan violencias interpersonales; las que son infligidas por otra persona o por un grupo pequeño de individuos (OMS, 2012), como el menoscabo, la exclusión, las descalificaciones, las ironías que toman lugar entre pares, o bien ante los malos tratos realizados por directivos. Este tipo de violencia interpersonal es preferentemente una violencia verbal, la que, de acuerdo a Galtung (1998), es una violencia visible. En la misma categoría se encuentran las violencias físicas, las cuales en los resultados alcanzados no se registran relaciones en las cuales se hayan manifestado. La Dictadura Militar, como realidad histórica y la idiosincrasia chilena se identifican como causas de violencias descritas en las relaciones interpersonales, configurándose como elementos basados en las violencias culturales que conviven en la escuela (Galtung, 
I998). En sintonía a lo planteado por Bourdieu (I998), hay violencia estructural ante la amenaza de desempleo y despido, la precariedad del trabajo docente anclado bajo las condiciones de resultados propias del neoliberalismo, situación graficada en la constante dependencia del factor matrícula. La violencia estructural también es evidenciada en la violencia de género que tiene lugar hacia figuras femeninas.

En coincidencia con la teoría, las violencias en el cotidiano de la escuela han sido naturalizadas y justificadas, situación que favorece su permanencia en el tiempo (Bringiotti, Krynveniuk y Lassi, 2007). Los resultados en las distintas dimensiones, como la carga horaria y la distribución del trabajo, las disminuidas remuneraciones, la necesidad de contar con otro trabajo, las normas laborales, por citar algunos de los elementos vistos, se posicionan como elementos que favorecen la intensificación y malestar en el trabajo docente. Ambos conceptos se pueden relacionar como manifestaciones de violencias, al afectar la integridad de la persona, en su organización y salud; en los distintos aspectos que la componen. El aspecto de intensificación es un concepto ya evidenciado por el gremio docente bajo el término agobio, sin embargo, el concepto malestar/bienestar docente es un área que se requiere hacer visible en la escuela como un ámbito de prevención de aspectos que afectan en la salud de quienes se insertan en la escuela como profesionales de la educación.

En esta oportunidad, el estudio se aboca a las violencias en las relaciones laborales de profesores y profesoras en distintos establecimientos educacionales de la Región Metropolitana urbana. Como aproximación a la etnografía, el estudio presentó limitantes en un ámbito práctico, al enfrentan dificultades de acceso al terreno. En el aspecto teórico se hace evidente lo planteado en las primeras páginas, sobre la multiplicidad de visiones que existen respecto al concepto de violencia. Este hecho afecta en el contraste con otras investigaciones que, si bien trabajan desde la violencia, no se sitúan desde las mismas concepciones.

A partir de la experiencia de etnografía, se visualizan violencias en el ámbito laboral en otros trabajadores de la escuela: quienes se desempeñan como asistentes de la educación. 
En su indispensable labor, estos actores identifican verticalidad en las relaciones con otros miembros, la constante crítica a su trabajo, el ser pasados a llevar por sus mismos colegas en las acciones que involucran a su rol, la relación con apoderados que exigen fuera de lo que implica su labor, por citar algunos elementos.

A partir de lo anterior, la psicología educacional cuenta con grandes desafíos pendientes: ¿cómo hablar de un buen trato en la comunidad escolar, cuando las condiciones en el ámbito laboral del profesorado pueden ser deficientes? ¿Cómo hacer frente en la escuela a la intensificación y malestar resultantes de las relaciones laborales actuales?

El estudio da cuenta de profesores cansados, con sobrecarga laboral, con una disminuida vida personal, en constante tensión, por nombrar algunos de los elementos que afectan su labor educativa y su relación con los demás actores. Quizás no hay un punto de inicio claro ante la panorámica de resultados evidenciados, pero sí es importante recalcar el rol de aquellos factores protectores que podrían contribuir positivamente en la escuela.

Ejemplo de ello es la significatividad del trabajo, las condiciones de remuneración o la visualización de las necesidades del profesorado en el cotidiano, como lo es el respetar y considerar su trabajo en la complejidad que ello implica como profesionales de la educación.

Finalmente, a través de este estudio, se da cuenta de las violencias en el trabajo de profesores y profesoras de la Región Metropolitana, por medio del concepto de relaciones laborales. Los resultados y hallazgos hacen patente que, para poder discutir sobre la mejora de la escuela en los aspectos que constantemente son evaluados como convivencia y enseñanza-aprendizaje, también se requiere poner sobre la mesa aspectos macros del trabajo docente. Si bien en las normativas -como políticas educativas- se encuentran presentes algunos de los factores evidenciados en este estudio, es necesario hacer el llamado respecto a la protección del trabajador de la educación, desde las instituciones que señalan la importancia del respeto a la integridad del profesorado. 


\section{Referencias bibliográficas}

Assael, J.; Cornejo, R.; González, J.; Redondo, J.; Sánchez, R. \& Sobarzo, M. (20II). La empresa educativa chilena. Educ. Soc., Campinas, 32 (II5), 305-322, abr.-jun.

Berger, C. y Lisboa, C. (2012). Violencia Escolar. Estudios y Posibilidades de Intervención en Latinoamérica. Santiago de Chile: Editorial Universitaria.

Blase, J. \& Blase, J. (2007). The mistreated teacher: a national study. Journal of Educational Administration, 46(3) 263 - 301.

Bourdieu, P. y Wacouant, L. (1995). Respuestas. Por una antropología reflexiva. México, D. F. Grijalbo.

Bourdieu, P (1998). La Esencia del Neoliberalismo. Le Monde.

Bringiotti, M.; Krynveniuk, M. y Lassi, S. (2007). Las múltiples violencias de la "violencia" en la escuela. Hacia un abordaje integrativo del problema. Paidéia, 2004, I4(29), 313-325.

Bruner, J. (1990). Actos de Significado, Más allá de la Revolución Cognitiva. Madrid: Alianza Editorial.

Cornejo, R. (2007). Entre el Sufrimiento Individual y los Sentidos Colectivos: Salud Laboral Docente y Condiciones de Trabajo. Docente, 35.

Cornejo, R.; Albornoz, N; Castañeda, L; Palacios, D.; Etcheberrigaray, R; Goméz, S.; Hidalgo, F. y Lagos, J. (2015). Las Prescripciones del Trabajo Docente en el Nuevo Marco Regulatorio de Políticas Educativas en Chile. Psicoperspectivas, 24(2), 72-83.

Debarbieux, E. (1996). La violence en milien scolaire. I- Etat des Iieux. Paris, Francia: ESF.

Debarbieux, E \& Blaya, C. (2009). Clia escolar i prevenció de la violencia. Educar. 43, 3I-4I.

Dubet, F. (2003). Las Figuras de la Violencia en la Escuela. Reflexiones Pedagógicas, Docencia, 19.

Dubet, F. (2005). La escuela de las Oportunidades. Barcelona: Gedisa.

García, M. y Madriaza, P. (2005). Sentido y Propósito de la violencia Escolar. Análisis Estructural del Discurso de Estudiantes Agresivos del Nivel Socioeconómico Alto. Revista de Psicología de la Universidad de Chile. Vol. XIV (I), 4I- 59.

Galtung, J. (1998). Tras la Violencia, 3 R: Reconstrucción, Reconciliación, Resolución. Afrontando Los Efectos Visibles e Invisibles de la Guerra y la Violencia. Bilbao, Colección Red Gernika. 
Geertz, C. (2005). La Interpretación de las Culturas. Barcelona: Gedisa.

Govea, V.; Vera, G. y Vargas, A. Etnografía: una mirada desde corpus teórico de la investigación cualitativa Omnia, l7(2), pp. 26-39.

Fernández, I.; Penacho, F. y Candelas, M. (20II). Reflexiones en Torno al Concepto de Violencias en las Aulas. Pulso, 34, 195-210.

Hargreaves, A. (2003). Profesorado, Cultura y Postmodernidad. Madrid: Morata.

Köhler, H. y Artiles, A. M. (2010). Manual de la Sociología del Trabajo y de las Relaciones Laborales. Madrid: Delta Publicaciones.

Ley 19. 070. Fija Texto Refundido, Coordinado y Sistematizado De La Ley N 19. 070 que Aprobó el Estatuto de los Profesionales de la Educación, y de las Leyes que la Complementan y Modifican. Biblioteca del Congreso Nacional. Santiago, Chile. 22 de enero de 1997.

Ley 20. 50I Calidad y Equidad de la Educación. Biblioteca del Congreso Nacional. Santiago, Chile. 201 I. 26 de febrero de 2011.

McMahon, S.; Martínez, A.; Espelage, D.; Rose, C. , Reddy, L.; Lane, K.; Anderman, E.; Reynolds, C.; Jones, A. \& Brown, V. (20I4). Violence Directed Against Teachers: Results From a National Survey. Psychology in the Schools, $5 \mathrm{l}(7)$.

Mineduc (2005). Principales Resultados del Estudio Nacional de Violencia en el Ámbito Escolar. Mineduc.

Mineduc (2013). Docentes en Cbile. Resultados de la Encuesta TALIS 2013. Extraído el I0 de octubre de 20I5, desde http:// centroestudios. mineduc. cl/ tp_enlaces/portales/tp5996f8b7cm96/uploadImg/File/Evidencias/ evidencia_final_marzo_20I5.pdf

Morales, M.; Álvarez, J. P.; Ayala, Á.; Ascorra, P.; Bilbao, M.; Carrasco, C.; López, V.; Olavarría, D.; Ortiz, S.; Urbina, C. y Villalobos, B. (2014). Violencia Escolar a Profesores: Conductas de Victimización Reportadas por Docentes de Enseñanza Básica. Revista de Estudios Cotidianos - NESOP, 2, 9I-II6.

Mucchelli, A. (1996). Diccionario de Métodos Cualitativos en Ciencias Humanas y Sociales. Madrid: Editorial Síntesis.

Ortiz-Molina, B. (20I I). Violencia escolar: la mirada de los maestros sobre las relaciones de colegaje. Magis, 4, 369-382.

Organización Mundial de la Salud (2002). Informe Mundial sobre la Violencia y la Salud. Washington, D.C., Organización Panamericana de la Salud, Oficina Regional para las Américas de la Organización Mundial de la Salud. 
Significados docentes sobre violencias en las relaciones laborales en la escuela - Barría et al.

UNESCO (2005). Condiciones de Trabajo y Salud Docente. Santiago de Chile: OREALC / UNESCO.

Valdivieso, P. (2009). Violencia escolar y relaciones intergrupales Sus prácticas y significados en las escuelas secundarias públicas de la comuna de Peñalolen en Santiago de Chile. Tesis doctoral, Universidad de Granada, Facultad de Psicología, Departamento de Psicología Social y Metodología de las Ciencias del Comportamiento.

Zerón, A. (2006). Sentido de la Violencia Escolar en Chile. Un Estudio de Sociología Comprensiva. Tesis para optar al grado de Doctorado en Ciencias de la Educación. Pontificia Universidad Católica de Chile. Santiago de Chile. 